hep-th/0404243

HUTP-04/A019, SU-ITP-04/16, SLAC-PUB-10425, TIFR/TH/04-11

\title{
On the Taxonomy of Flux Vacua
}

\author{
Alexander Giryavets ${ }^{a * 1}$, Shamit Kachru ${ }^{a 2}$ and Prasanta K. Tripathy ${ }^{b, c 3}$ \\ ${ }^{a}$ Department of Physics and SLAC \\ Stanford University \\ Stanford, CA 94305/94309 USA \\ $b$ Tata Institute for Fundamental Research \\ Homi Bhabha Road, Mumbai 400 005, INDIA \\ c Jefferson Laboratory \\ Harvard University \\ Cambridge, MA 02138 USA
}

We investigate several predictions about the properties of IIB flux vacua on Calabi-Yau orientifolds, by constructing and characterizing a very large set of vacua in a specific example, an orientifold of the Calabi-Yau hypersurface in $W P_{1,1,1,1,4}^{4}$. We find support for the prediction of Ashok and Douglas that the density of vacua on moduli space is governed by $\operatorname{det}(-R-\omega)$ where $R$ and $\omega$ are curvature and Kähler forms on the moduli space. The conifold point $\psi=1$ on moduli space therefore serves as an attractor, with a significant fraction of the flux vacua contained in a small neighborhood surrounding $\psi=1$. We also study the functional dependence of the number of flux vacua on the D3 charge in the fluxes, finding simple power law growth.

April 2004

\footnotetext{
* On leave from Steklov Mathematical Institute, Moscow, Russia

1 giryav@stanford.edu

2 skachru@stanford.edu

3 prasanta@theory.tifr.res.in

Work supported in part by the Department of Energy Contract DE-AC03-76SF00515

Stanford Linear Accelerator Center, Stanford University, Stanford, CA 94309
} 


\section{Introduction}

Since the classic work of Candelas, Horowitz, Strominger and Witten in the mid 1980s [1], Calabi-Yau compactification of 10d supergravity has been the dominant paradigm for the construction of $4 \mathrm{~d}$ string models. In the heterotic string theory, such compactifications yield $\mathcal{N}=1$ supersymmetric theories below the KK scale, and can quite plausibly accomodate the standard model. In the type II context, Calabi-Yau compactification yields $\mathcal{N}=2$ supersymmetric models, which have been of considerable interest in studies of string duality and mirror symmetry.

More recently, it has become clear that these models are only the simplest representatives of a much larger class of models. For concreteness, we will restrict our discussion to the type IIB theory, but similar remarks would apply in the other limits of M-theory. In the IIB context, the theory contains p-form gauge fields which couple to the various supersymmetric D-branes and NS-branes. One can consider magnetic fluxes of these gauge fields in the internal dimensions; such fluxes are consistent with 4d Lorentz invariance. In fact, in type II orientifold models which break the supersymmetry from $\mathcal{N}=2$ to $\mathcal{N}=1$, generic methods of satisfying the tadpole conditions will involve the addition of background magnetic flux. This is interesting because the magnetic fluxes will cost an energy which depends on the detailed shape and size of the internal dimensions; that is, they will generate a potential for some of the Calabi-Yau moduli.

In the case of type IIB on a Calabi-Yau orientifold, the allowed fluxes are those of the RR and NS three-form field strengths $F_{3}$ and $H_{3}$. The properties of the theory in the presence of such fluxes have been investigated in a vast literature; see for instance [2-17]. The resulting $\mathcal{N}=1$ supergravity theories (derived in [4, 8]) are of the no-scale type, with a Kähler potential $K$ inherited (at large volume and weak coupling) from the CalabiYau compactification and a superpotential which is computable in terms of the choice of quantized background magnetic fluxes [3]. The resulting scalar potential generically fixes the complex structure moduli and the dilaton. It is possible to make semi-realistic chiral models of particle physics in this framework [9], and to describe some aspects of the resulting supergravity theories in the general framework of gauged extended supergravity [10]. The theory of soft susy breaking terms in these models has been developed in [11].

For a given choice of the integral background fluxes, the superpotential for moduli can simply be written as a combination of periods of the holomorphic three-form $\Omega$ on the Calabi-Yau space. The periods are computable by standard techniques in classical 
geometry, highly developed in the physics literature after the seminal work of Candelas et al on mirror symmetry [12]. The resulting superpotential is exact at least to all orders in the $\alpha^{\prime}$ expansion by standard holomorphy arguments. It can be argued that "generic" nonperturbative effects (known to occur in various models in various circumstances, and likely to occur in a generic setting due to the absence of symmetries that would prevent them) should suffice to fix the remaining (Kähler) moduli in some cases [13, 14]. As argued in [13], if the SUSY-breaking ( $e^{K}|W|^{2}$ evaluated in vacuum) by the fluxes is suitably small, and if $g_{s}$ is stabilized by the fluxes at weak coupling, one can have small expansion parameters which allow one to stabilize the remaining moduli in a regime of control. Of course, this will only happen in at most a small fraction of the vacua. As emphasized in [13], following the basic ideas of Bousso and Polchinski [15](see also [16]), the large number of possible integral flux choices makes it plausible that suitable flux choices do exist, which allow one to fix $g_{s}$ at weak coupling and keep the SUSY breaking hierarchically smaller than the string scale.

This set of issues, which goes into establishing the existence of a "landscape" of string/M-theory vacua (like the "discretuum" of [15]) and characterizing its properties, can and should be studied systematically and quantitatively. In addition to the works already described, a persuasive discussion of this can be found in the works of Douglas and collaborators [14,17,19] (see also [20,21] for more general comments about the landscape). In particular, in [17], under well motivated assumptions some theorems are proven regarding the numbers and distribution of flux vacua on the Calabi-Yau moduli space (really, the moduli space of complex structures and dilaton VEVs) $\mathcal{M}$. The assumption which goes into proving these theorems is, roughly, that the superpotential is a random section (with respect to a covariance kernel determined by the Kähler potential) of the appropriate line bundle over $\mathcal{M}$. This models the flux superpotentials as random combinations of periods with respect to some measure, instead of as the precise integral combinations of periods determined by various choices of $H_{3}$ and $F_{3}$ consistent with the tadpole conditions. Basically, this assumption follows from the statement that if one allows large enough fluxes, flux quantization becomes irrelevant for the average properties of the vacua. The natural size of the fluxes required by tadpole conditions in IIB string theory probably falls into this range.

Because the emerging discretuum of vacua is too complicated to allow us to usefully examine each vacuum individually, this kind of program to find simple assumptions which accurately characterize the space of vacua seems to us rather important. In fact, following 
«17,19], one could simply disregard the Kähler moduli altogether, ignore the no-scale structure (which is certainly broken by quantum corrections), and characterize the properties of the supergravities that result with the given $K$ and $W$ as a function of complex structure moduli. The resulting ensemble of supergravity theories is rich enough that it is very plausible that some aspects of the full story (about the real space of string vacua), are captured by this simplified ensemble. However, we will continue to use the language and equations appropriate to thinking of these as vacua which arise in the no-scale approximation to the full IIB theory, though it is easy to translate back and forth.

In this paper, we test the prediction of [17 regarding the distribution of vacua on $\mathcal{M}$, by studying the vacua which result on a subspace of the moduli space of a certain Calabi-Yau threefold, in the presence of a small subset of the possible fluxes. While we are looking at a very small fraction of the possible fluxes and hence of the total number of flux vacua on this space, the numbers even on our subspace are easily large enough to allow a meaningful test of the conjecture. In addition, in these vacua there is a tadpole condition that requires $\int H_{3} \wedge F_{3}=N_{\text {flux }} \leq L$, where $L$ is some number fixed by global details of the model. The growth of the number of vacua with $L$, fixing the number of cycles which support flux, is subject to various conjectures along the lines of [15]. Here, in addition to testing the distribution of vacua on moduli space, we also test the growth of the number of vacua as a function of $L$, and find a simple power law. A proper understanding of the theory which determines this power is rather complicated, as we describe in the following; some relevant results are discussed in the revised version of [19].

The organization of this paper is as follows. In $\S 2$, we describe the predictions we wish to test. In $\S 3$, we discuss the model in which we will test them (which is "model A" of [7]). This model has (on the locus of interest to us) a one-dimensional complex structure moduli space, parametrized by a complex variable $\psi$. In sections 4 and 5 , we describe how we find solutions of the flux equations in two regions in the moduli space, using expansions of the periods around $\psi=0$ (the mirror Landau-Ginzburg point) and $\psi=1$ (the conifold point). In each of these cases, we find sufficiently large numbers of vacua to perform meaningful tests of the predictions. In $\S 6$ we present our conclusions.

We should stress that although the discussion here is specific to type IIB vacua of the class described in [4] (for which several de Sitter scenarios exist [13,22]), a similar landscape also emerges from careful consideration of the various other limits of M-theory (see e.g. 223] for discussions in the heterotic setting, and [24] for an early construction of de Sitter vacua in noncritical string theories). For instance in the heterotic string, one needs to consider 
the large class of possible gauge bundles in each $E_{8}$, in addition to the possibility of NS three-form flux and Non-Kählerity [25]. This is technically more challenging than studying the class of IIB flux vacua we considered here, but finding similar tractable toy ensembles in that setting should be possible. Another setting where similar equations arise is in the problem of finding BPS black holes in $\mathcal{N}=2$ supergravity; the interesting connections between that problem and the problem of finding flux vacua are described in [26].

While this work was being completed, the paper [19] which has some overlap with our results appeared.

\section{The conjectures}

We will be interested in testing two conjectures about the large class of IIB flux vacua described in [4], in the no-scale approximation. The conjectures concern the distribution of flux vacua on the moduli space of complex structures, and the scaling of the number of flux vacua with the D3-charge in the fluxes. They can be proven to hold [17] under suitable assumptions about the irrelevance of flux quantization, so we are really testing whether those assumptions hold for simple representatives of the class of vacua described in $[4$.

\subsection{Where do the vacua lie?}

In a recent paper of Ashok and Douglas [17, it was conjectured that the index density of the no-scale vacua described in [4] is given by an integral

$$
\mathcal{I} \sim \int_{\mathcal{M}} \operatorname{det}(-R-\omega) .
$$

$\mathcal{I}$ is normalized appropriately to the total number (or index) of no-scale vacua. Here $\omega$ is the Kähler form on the moduli space and $R$ is the curvature two-form expressed as a Hermitian matrix

$$
R_{k}^{l}=R_{i \bar{j} k}^{l} d z^{i} \wedge d \bar{z}^{\bar{j}}
$$

with $z^{i}$ the coordinates on $\mathcal{M}$. The moduli space $\mathcal{M}$ consists of the fundamental domain of any relevant duality groups, acting on the complex structure and axio-dilaton Teichmuller spaces. In our example, it will be a product of the standard fundamental domain of $S L(2, Z)$ in the upper half-plane (for the axio-dilaton $\tau$ ) with a (complex) one-dimensional slice of a Calabi-Yau complex structure moduli space. As described in [17,19, it is possible 
to interpret the conjecture as a statement about the distribution of vacua on the CalabiYau complex structure moduli space (forgetting about the axio-dilaton VEV). Therefore, in all of our results, we will describe the distribution of vacua on the Calabi-Yau moduli space, ignoring the value of the axio-dilaton in the solution (i.e. for solutions distributed all over $\tau$-space but in some fixed ball in complex structure moduli space).

We will assume that there are no miraculous cancellations, so that the total number of vacua scales like $\mathcal{I}$. In this case, one predicts that the number of vacua in a given region of complex structure moduli space should scale like $\operatorname{det}(-R-\omega)$, where $R$ and $\omega$ are now interpreted as the curvature and Kähler form just on the complex structure moduli space. We will test this conjecture in sections 4 and 5 by computing the numbers of vacua lying in various regions of the moduli space (by using monte carlo simulations to choose fluxes and then solving the flux equations), and checking that they are distributed like the appropriate integrals of $\operatorname{det}(-R-\omega)$.

\subsection{Growth of number of vacua with $L$}

The three-form fluxes in type IIB supergravity contribute to the D3-brane charge tadpole on the compact space, via an equation of the form

$$
N_{\text {flux }}=\frac{1}{(2 \pi)^{4}\left(\alpha^{\prime}\right)^{2}} \int_{M} H_{3} \wedge F_{3}
$$

where tadpole cancellation requires

$$
N_{\text {D3 }}+N_{\text {flux }}=L
$$

Here $L$ is some number fixed by the orientifold charge in the given model and $N_{\mathrm{D} 3}$ is the number of space-time filling D3 branes, transverse to $M$.

While it may appear from (2.4) that this is not much of a restriction, since the quadratic form $\int H_{3} \wedge F_{3}$ is not positive definite, this is not quite correct. On solutions of the equations of motion, one finds (as on p.4 of the first reference in [5]) that $F_{3} \sim-*_{6} H_{3}$, so $N_{\text {flux }}$ becomes a positive semidefinite form. This means that in a space with $K$ three-cycles, one might heuristically think of (2.4) as allowing one to search for flux vacua at each integral lattice point within a sphere of radius $\sqrt{L}$ in flux space. Although $H$ and $F$ together involve $2 \mathrm{~K}$ flux integers, using the relation between $H$ and $F$ on solutions indicates that for a fixed point in moduli space, this will be a $K-1$ sphere (instead of a $2 K-1$ sphere). So for e.g. a $\left(T^{2}\right)^{3}$ with square two-tori, one can easily rewrite (2.4) 
(setting $N_{D 3}=0$ ) as the equation for a sphere in $K$ dimensions. Then, the number of solutions for the flux equations $N_{\text {vacua }}$, like the number of lattice points inside this sphere, presumably scales like

$$
N_{\text {vacua }} \sim L^{K / 2}
$$

This would be the behavior of the number of solutions, in the simple toroidal example above, at a point in $\mathcal{M}$ where solutions to the flux equations exist. 1

This is the kind of logic suggested in e.g. [15], applied in this context by many workers [28], and rigorously argued to hold in this context in [17]. However, the results of [17] contain a surprise - the predicted scaling of the total number of vacua is actually

$$
N_{\text {vacua }} \sim L^{K}
$$

instead of the $L^{K / 2}$ in (2.5). To understand this, average of the number of solutions per unit volume in $\mathcal{M}$ - the number of solutions jumps discontinuously as one moves in $\mathcal{M}$, so one must average to get a reasonable formula. This averaging produces an enhancement of the result - for large enough $L$, a given small region of moduli space will give rise to $L^{K}$ lattice points within flux space, instead of the naive $L^{K / 2}$. This in particular means that if one looks at finite $L$ in some region of the moduli space, one may find an intermediate scaling that is between the naive prediction (2.5) and the $L^{K}$ behavior (2.6) expected from [17]. The detailed crossover behavior determining how the scaling should go for a given region in $\mathcal{M}$ at various $L$ and $K$ is a complicated subject which is still under investigation (see the revised version of the paper by Denef and Douglas [19] for further discussion).

Because of the complications described above, we simply take our results as an experimental clue that should help in the development of the full theory of how $N_{\text {vacua }}$ scales with $L$ and $K$, though we expect to (and do) find a power intermediate between (2.5) and (2.6).

1 One might ask whether one could increase the number of solutions by including anti-D3 branes (i.e. taking $N_{D 3}<0$ in (2.4)). While one can include anti-branes in these models, the number is bounded by the decay mechanism discussed in [27], and we will simply assume $N_{D 3} \geq 0$ for now.

2 We are grateful to M. Douglas for several discussions about this subject. 


\section{The model of interest}

We will study an orientifold of the Calabi-Yau threefold $M$ which arises as a hypersurface in the weighted projective space $W P_{1,1,1,1,4}^{4}$

$$
4 x_{0}^{2}+x_{1}^{8}+x_{2}^{8}+x_{3}^{8}+x_{4}^{8}-8 \psi x_{0} x_{1} x_{2} x_{3} x_{4}=0 .
$$

This threefold has $h^{1,1}(M)=1$ and $h^{2,1}(M)=149$. As explained in [7], an orientifold of $M$ arises as a limit of the F-theory compactification on the Calabi-Yau fourfold $X$ given by a hypersurface in $W P_{1,1,1,1,8,12}^{5}$. The orientifold action takes $x_{0} \rightarrow-x_{0}$ and $\psi \rightarrow-\psi$ (in conjunction with the worldsheet parity reversal), while leaving all other variables unchanged. This limit of the F-theory model on $X$ is obtained by following the general prescription given by Sen [29]. One of the benefits of knowing the F-theory lift is that it allows us to easily compute the tadpole condition for compactification on the orientifold of $M$. The relevant condition is (see [30] and the third paper listed in [2])

$$
N_{\text {D3 }}+N_{\text {flux }}=\frac{\chi(X)}{24}=972
$$

where $N_{\mathrm{D} 3}$ is the number of space-filling D3 branes one inserts transverse to $M$, and $N_{\text {flux }}$ is the amount of D3 charge carried by the $H_{3}$ and $F_{3}$ fluxes as given in (2.3).

In general, one could add many deformations to the defining equation (3.1) which are consistent with the orientifold action. However, as explained in [7] following [31], the equation (3.1) is the most general equation consistent with a global symmetry group $\mathcal{G}=Z_{8}^{2} \times Z_{2}$ acting on the projective coordinates. If one turns on only fluxes which are consistent with preserving this symmetry, then the resulting superpotential is guaranteed to depend on the additional moduli only at higher orders (in $\mathcal{G}$ invariant combinations). This means that if we set these moduli to zero (as done in (3.1)), compute the periods relevant for our subset of fluxes, and solve the resulting equations for $\psi$ and the axiodilaton $\tau$, we are guaranteed that the solutions we find will be solutions of the full theory. The additional moduli can be consistently set to zero because of the symmetry, and will generically be constrained by a higher order potential in the resulting vacua.

In fact, the subset of periods which are relevant to the $\mathcal{G}$ invariant fluxes coincide (up to a factor of $|\mathcal{G}|$ ) with those of the mirror manifold to $M, W$, as explained in [7]. These correspond to periods on four different 3-cycles in $M$, and hence we proceed to develop the relevant formalism for computing flux superpotentials involving only $H_{3}$ and $F_{3}$ fluxes 
through these four 3-cycles. Happily, the periods of interest to us in this example were computed in one of the early studies of mirror symmetry, by Klemm and Theisen [32].

In comparing to the conjecture of $\S 2.2$, we see that our problem has $K=4$ and $L=972$. So in the physical problem of interest, we could check the number of vacua with flux charge $N_{\text {flux }} \leq L_{*}$ for any $L_{*} \leq 972$. (Of course formally, we are free to consider vacua using the periods from this model with arbitrary $L$ ).

\subsection{Homology and Cohomology bases}

We will work with a symplectic homology basis for the subspaces of $H_{3}$ of interest to us. The basis of three-cycles $A_{a}$ and $B^{a}(a=1,2)$ and the basis for integral cohomology $\alpha_{a}$ and $\beta^{a}$ satisfy

$$
\int_{A_{a}} \alpha_{b}=\delta_{b}^{a}, \quad \int_{B^{b}} \beta^{a}=-\delta_{b}^{a}, \quad \int_{M} \alpha_{a} \wedge \beta^{b}=\delta_{a}^{b} .
$$

The holomorphic three form can be represented in terms of periods in this basis as follows:

$$
\int_{A_{a}} \Omega=z^{a}, \quad \int_{B^{a}} \Omega=\mathcal{G}_{a}, \quad \Omega=z^{a} \alpha_{a}-\mathcal{G}_{a} \beta^{a} .
$$

In addition

$$
\int_{M} \Omega \wedge \bar{\Omega}=\bar{z}^{a} \mathcal{G}_{a}-z^{a} \overline{\mathcal{G}}_{a}=\bar{z}^{a} \frac{\partial \mathcal{G}}{\partial z^{a}}-z^{a} \frac{\partial \overline{\mathcal{G}}}{\partial \bar{z}^{a}}=-\Pi^{\dagger} \cdot \Sigma \cdot \Pi .
$$

Here, we have introduced the prepotential $\mathcal{G}\left(z^{1}, z^{2}\right)$, the period vector $\Pi$ (whose entries are the periods $(3.4))$, and the matrix

$$
\Sigma=\left(\begin{array}{cc}
0 & 1 \\
-1 & 0
\end{array}\right)
$$

whose entries are two by two matrices.

\subsection{Fluxes, Superpotential and Kähler Potential}

The NS and RR fluxes admit the following quantization condition

$$
F_{(3)}=(2 \pi)^{2} \alpha^{\prime}\left(f_{a}\left[B_{a}\right]+f_{a+2}\left[A_{a}\right]\right), \quad H_{(3)}=(2 \pi)^{2} \alpha^{\prime}\left(h_{a}\left[B_{a}\right]+h_{a+2}\left[A_{a}\right]\right)
$$

with integer $f_{i}$ and $h_{i}$. Here we used the notation $\left[A_{a}\right]=\alpha_{a}$ and $\left[B_{a}\right]=\beta_{a}$. Using this notation, we find the following expression for $N_{\text {flux }}$

$$
N_{\text {flux }}=\frac{1}{(2 \pi)^{4}\left(\alpha^{\prime}\right)^{2}} \int_{M} H_{(3)} \wedge F_{(3)}=f^{T} \cdot \Sigma \cdot h .
$$


The superpotential is given by

$$
W=\int_{M}\left(F_{(3)}-\tau H_{(3)}\right) \wedge \Omega=(2 \pi)^{2} \alpha^{\prime}(f \cdot \Pi-\tau h \cdot \Pi) .
$$

The Kähler potential for the dilaton-axion and complex moduli is given by

$$
K=K_{\tau}+K_{\psi}=-\ln (-i(\tau-\bar{\tau}))-\ln \left(i \int_{M} \Omega \wedge \bar{\Omega}\right)=-\ln (-i(\tau-\bar{\tau}))-\ln \left(-i \Pi^{\dagger} \cdot \Sigma \cdot \Pi\right)
$$

\subsection{Conditions for solutions}

The tadpole condition for the model of interest is given by (3.2). This provides a restriction on the fluxes we can use, i.e. on our allowed class of superpotentials - we must keep $N_{\text {flux }} \leq 972$.

We will be searching for nonsupersymmetric flux vacua which obey the equations

$$
D_{\tau} W=0 \quad \text { and } \quad D_{\psi} W=0
$$

They are nonsupersymmetric because in the full IIB string theory, they will generically have $D_{\rho} W \sim W \neq 0$ where $\rho$ is a Kähler modulus. Note however that in the toy ensemble of [17,19, such vacua are called supersymmetric, since the Kähler moduli are not part of the toy ensemble.

\section{Small $\psi$ region}

\subsection{Periods, Kähler potential, Metric and Curvature form}

The periods for the small $\psi,|\psi|<1$ region are given by

$$
w_{j}(\psi)=(2 \pi i)^{3} \frac{\pi}{8} \sum_{n=1}^{\infty} \frac{1}{\Gamma(n)\left(\Gamma\left(1-\frac{n}{8}\right)\right)^{4} \Gamma\left(1-\frac{n}{2}\right)} \frac{\exp \left(\frac{7 \pi i}{8} n\right)}{\sin \left(\frac{\pi n}{8}\right)} \frac{\left(4 \alpha^{j} \psi\right)^{n}}{\psi}
$$

where $\alpha=\exp \left(\frac{\pi i}{4}\right)$. The corresponding period vector is given by

$$
w^{T}=\left(w_{2}(\psi), w_{1}(\psi), w_{0}(\psi), w_{7}(\psi)\right)
$$

We will want the periods in a symplectic basis. In such a basis, the period vector $\Pi^{T}(\psi)=$ $\left(\mathcal{G}_{1}(\psi), \mathcal{G}_{2}(\psi), z^{1}(\psi), z^{2}(\psi)\right)$ is provided by the linear transformation

$$
\Pi=m \cdot w
$$


where the matrix $m$ is given by

$$
m=\left(\begin{array}{cccc}
-\frac{1}{2} & -\frac{1}{2} & \frac{1}{2} & \frac{1}{2} \\
0 & 0 & -1 & 0 \\
-1 & 0 & 3 & 2 \\
0 & 1 & -1 & 0
\end{array}\right)
$$

The period vector in the symplectic basis can be written in the form

$$
\Pi(\psi)=\sum_{n=0}^{\infty} c_{2 n} p_{2 n} \psi^{2 n}
$$

Here the vectors $p_{2 n}$ are given by

$$
p_{2 n}=m \cdot\left(\alpha^{2(2 n+1)}, \alpha^{(2 n+1)}, 1, \alpha^{7(2 n+1)}\right)^{T}
$$

and the first few constants $c_{2 n}$ of interest are given by

$$
\begin{array}{ll}
c_{0}=(2 \pi i)^{3} \frac{\sqrt{\pi}}{2 \Gamma^{4}(7 / 8)} \frac{\exp \left(\frac{7 \pi i}{8}\right)}{\sin \left(\frac{\pi}{8}\right)}, & c_{2}=-(2 \pi i)^{3} \frac{2 \sqrt{\pi}}{\Gamma^{4}(5 / 8)} \frac{\exp \left(\frac{5 \pi i}{8}\right)}{\sin \left(\frac{3 \pi}{8}\right)}, \\
c_{4}=(2 \pi i)^{3} \frac{4 \sqrt{\pi}}{\Gamma^{4}(3 / 8)} \frac{\exp \left(\frac{3 \pi i}{8}\right)}{\sin \left(\frac{5 \pi}{8}\right)}, & c_{8}=(2 \pi i)^{3} \frac{\sqrt{\pi}}{768 \Gamma^{4}(7 / 8)} \frac{\exp \left(\frac{7 \pi i}{8}\right)}{\sin \left(\frac{\pi}{8}\right)} .
\end{array}
$$

This yields the following Kähler potential for the complex structure moduli space:

$$
K_{\psi}=-\ln \left[\lambda_{0}+\lambda_{2}|\psi|^{4}+\lambda_{4}|\psi|^{8}+\lambda_{5} \psi^{8}+\bar{\lambda}_{5} \bar{\psi}^{8}+O\left(|\psi|^{12}\right)\right]
$$

where the real constants are given by

$$
\begin{array}{ll}
\lambda_{0}=-i\left|c_{0}\right|^{2} p_{0}^{\dagger} \cdot \Sigma \cdot p_{0}, & \lambda_{2}=-i\left|c_{2}\right|^{2} p_{2}^{\dagger} \cdot \Sigma \cdot p_{2}, \\
\lambda_{4}=-i\left|c_{4}\right|^{2} p_{4}^{\dagger} \cdot \Sigma \cdot p_{4}, & \lambda_{5}=-i \bar{c}_{0} c_{8} p_{0}^{\dagger} \cdot \Sigma \cdot p_{0} .
\end{array}
$$

Note that $p_{0}^{\dagger} \cdot \Sigma \cdot p_{0}=2 i(\sqrt{2}+2)$ and $p_{2}^{\dagger} \cdot \Sigma \cdot p_{2}=-p_{4}^{\dagger} \cdot \Sigma \cdot p_{4}=2 i(\sqrt{2}-2)$.

The resulting Kähler metric takes the form

$$
g_{\psi \bar{\psi}}=-4 \frac{\lambda_{2}}{\lambda_{0}}|\psi|^{2}\left(1+\left(4 \frac{\lambda_{4}}{\lambda_{2}}-2 \frac{\lambda_{2}}{\lambda_{0}}\right)|\psi|^{4}+O\left(\psi^{8}\right)\right) .
$$

Using the expression for the curvature form

$$
R_{\psi \bar{\psi}}=R_{\psi \bar{\psi} \psi}^{\psi}=-\partial_{\psi} \partial_{\bar{\psi}} \ln \left(g_{\psi \bar{\psi}}\right)
$$

one finds the following expression of interest

$$
R_{\psi \bar{\psi}}=-4|\psi|^{2}\left(4 \frac{\lambda_{4}}{\lambda_{2}}-2 \frac{\lambda_{2}}{\lambda_{0}}\right)+O\left(|\psi|^{6}\right)
$$

Note also that one finds

$$
\partial_{\psi} K_{\psi}=-2 \frac{\lambda_{2}}{\lambda_{0}}|\psi|^{2} \bar{\psi}+O\left(\psi^{7}\right)
$$

which is of use in evaluating Kähler covariantized derivatives with respect to $\psi$. 


\subsection{Distribution of flux vacua}

Given these facts about the Calabi-Yau moduli space geometry, it is now possible to work out the detailed prediction from $§ 2.1$. One sees that

$$
\int_{\mathcal{M}} \operatorname{det}(-R-\omega)=\mathrm{const} \int_{0}^{r}|\psi|^{2}|\psi| d|\psi|=\mathrm{const} \frac{r^{4}}{4}
$$

for (an eight-fold cover of) a small piece of the fundamental region of complex structure moduli space $\mathcal{M}:|\psi|<r$.

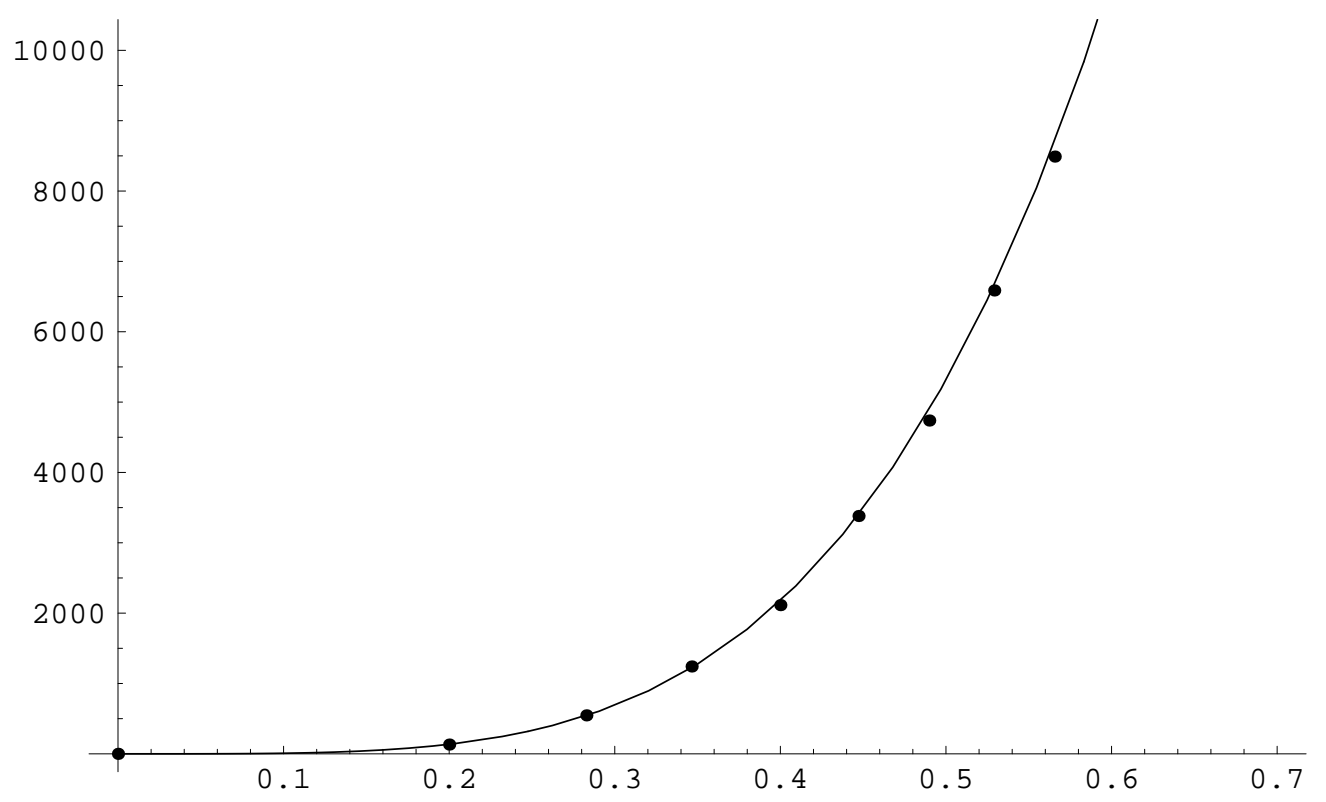

Fig. 1: A plot of a numerical evaluation of $\int_{\mathcal{M}} \operatorname{det}(-R-\omega)$. The monte carlo simulation data for each point is: number of random fluxes $N=10^{8}$; random flux interval $f, h \in(-40,40)$. The data is fit by the curve $340000 \frac{r^{4}}{4}$.

The equations for flux vacua, keeping terms up to order $\psi^{2}$, become

$$
\begin{aligned}
& D_{\tau} W=0 \quad \Rightarrow \quad(f-\bar{\tau} h) \cdot\left(c_{0} p_{0}+c_{2} p_{2} \psi^{2}\right)=0 \\
& D_{\psi} W=0 \quad \Rightarrow \quad(f-\tau h) \cdot\left(c_{2} p_{2}-\frac{\lambda_{2}}{\lambda_{0}} c_{0} p_{0} \bar{\psi}^{2}\right)=0 .
\end{aligned}
$$

This provides two equations

$$
\psi^{2}=-\frac{c_{0}}{c_{2}} \frac{(f-\bar{\tau} h) \cdot p_{0}}{(f-\bar{\tau} h) \cdot p_{2}}=\frac{c_{0}}{c_{2}} \frac{p_{0}^{\dagger} \cdot \Sigma \cdot p_{0}}{p_{2}^{\dagger} \cdot \Sigma \cdot p_{2}} \frac{(f-\bar{\tau} h) \cdot p_{2}^{\dagger}}{(f-\bar{\tau} h) \cdot p_{0}^{\dagger}} .
$$


The last one gives a quadratic equation for $\tau$ such that

$$
a \bar{\tau}^{2}-b \bar{\tau}+c=0 \quad \bar{\tau}=\frac{b \pm \sqrt{b^{2}-4 a c}}{2 a},
$$

where we defined the following quantities depending on fluxes

$$
\begin{aligned}
& \kappa=\frac{p_{0}^{\dagger} \cdot \Sigma \cdot p_{0}}{p_{2}^{\dagger} \cdot \Sigma \cdot p_{2}}=\frac{1+\sqrt{2}}{1-\sqrt{2}} ; \\
& a=\left|h \cdot p_{0}\right|^{2}+\kappa\left|h \cdot p_{2}\right|^{2} ; \\
& b=\left(h \cdot p_{0}\right)\left(f \cdot p_{0}^{\dagger}\right)+\left(f \cdot p_{0}\right)\left(h \cdot p_{0}^{\dagger}\right)+\kappa\left(\left(h \cdot p_{2}\right)\left(f \cdot p_{2}^{\dagger}\right)+\left(f \cdot p_{2}\right)\left(h \cdot p_{2}^{\dagger}\right)\right) ; \\
& c=\left|f \cdot p_{0}\right|^{2}+\kappa\left|f \cdot p_{2}\right|^{2} .
\end{aligned}
$$

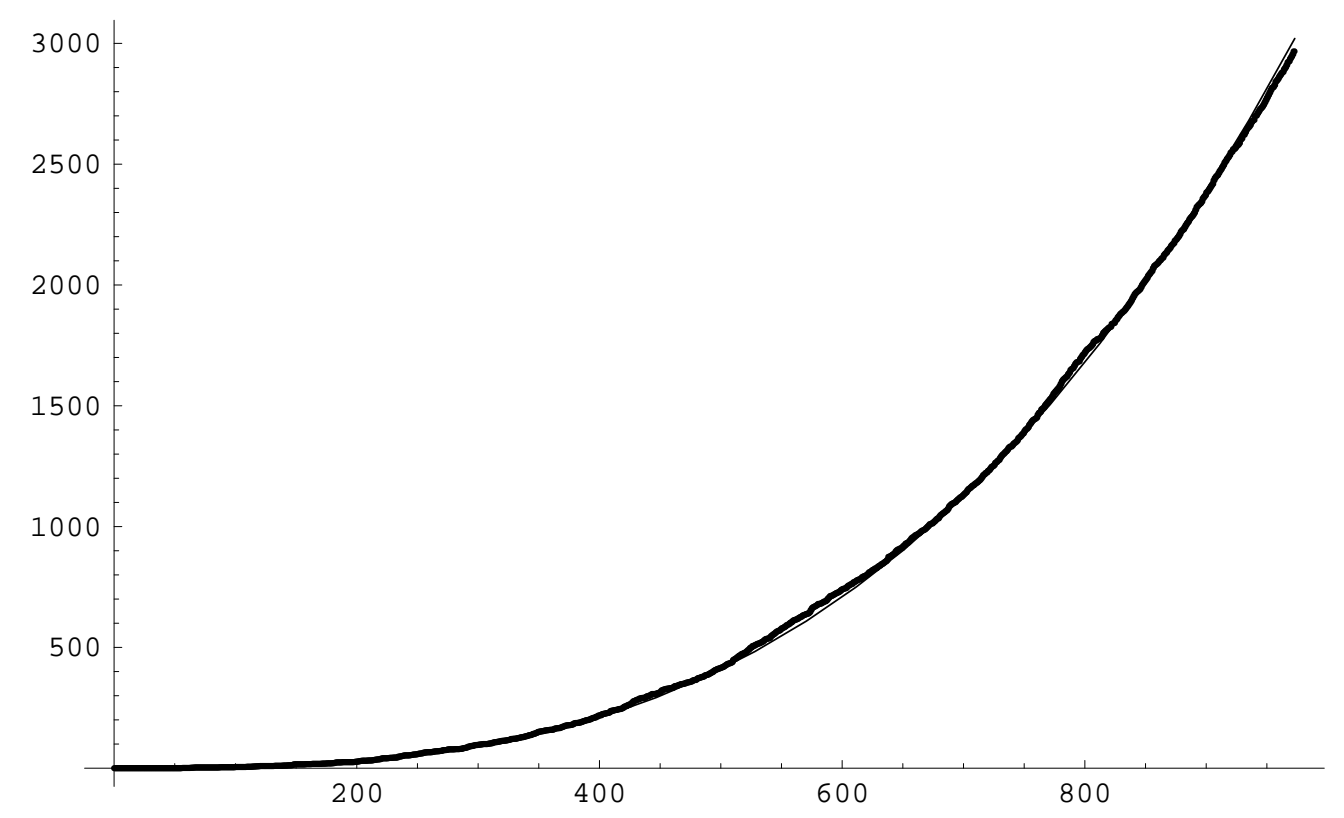

Fig. 2: The numerical results for the number of vacua with $N_{\text {flux }}<L$ for $L \in$ $(1,972)$. The monte carlo simulation data is: number of random fluxes $N=10^{10}$; random flux interval $f, h \in(-100,100)$; complex structure $\psi$ space region $|\psi|^{2}<$ 0.5 . The data is fit by the curve $\frac{L^{3}}{305000}$.

We used monte carlo simulations to check the validity of the formula (4.14) and the scaling of the number of vacua with $L$. We chose correspondingly $10^{8}$ and $10^{10}$ random fluxes, taking integer values $f, h$ in the ranges indicated in the figure captions. The number of solutions with $L \leq 972$ resulting in small $\psi$ is determined numerically from eqs. (4.16)(4.18) and is plotted in Fig.1. Fig.2 shows the dependence of the total number of vacua on $L$. 
One sees that the curve in Fig. 1 is in very good agreement with the prediction (2.1). It is interesting that in Fig. 2, one sees a rather clean $L^{3}$ growth of the number of vacua. The naive formula (2.5) would predict $L^{2}$, while the more refined formula proved by Ashok and Douglas under certain assumptions would predict $L^{4}$. In fact, as described in $\S 2.2$ and the revised version of [19], the correct scaling for a small region in $\psi$-space is a complicated subject. It may be that exceedingly large $L$ would be required to attain the scaling (2.6), in this region of moduli space.

\section{Conifold region}

\subsection{Periods, Kähler potential, Metric and Curvature form}

The periods in a symplectic basis in the vicinity of the conifold point $\psi=1$ can be given to the first order by the following expressions (here $x \equiv 1-\psi$ and $|x| \ll 1$ )

$$
\begin{aligned}
\mathcal{G}_{1}(x) & =(2 \pi i)^{3}\left[a_{0}+a_{1} x+O\left(x^{2}\right)\right], \\
\mathcal{G}_{2}(x) & =\frac{z^{2}(x)}{2 \pi i} \ln (x)+(2 \pi i)^{3}\left[b_{0}+b_{1} x+O\left(x^{2}\right)\right], \\
z^{1}(x) & =(2 \pi i)^{3}\left[c_{0}+c_{1} x+O\left(x^{2}\right)\right], \\
z^{2}(x) & =(2 \pi i)^{3}\left[d_{0}+d_{1} x+O\left(x^{2}\right)\right] .
\end{aligned}
$$

where the constants can be approximated by the following numbers

$$
\begin{array}{ll}
a_{0}=-1.774 i, & c_{0}=4.952-5.321 i, \\
a_{1}=1.227 i, & c_{1}=-4.488+3.682 i, \\
b_{0}=-1.047, & d_{0}=0, \\
b_{1}=0.451+0.900 i, & d_{1}=1.800 i .
\end{array}
$$

The Kähler potential for complex structure modulus is given by

$$
K_{\psi}=-\ln \left[\mu_{0}+\mu_{1} x+\bar{\mu}_{1} \bar{x}+\mu_{2}|x|^{2} \ln |x|^{2}+\mu_{3}|x|^{2}+\mu_{4} x^{2}+\bar{\mu}_{4} \bar{x}^{2}+O\left(|x|^{3} \ln |x|\right)\right],
$$

where the relevant constants $\mu_{0}, \mu_{1}, \mu_{2}$ and $\mu_{3}$ are given by

$$
\begin{array}{ll}
\mu_{0}=i(2 \pi)^{6}\left(a_{0} \bar{c}_{0}-c_{0} \bar{a}_{0}\right), & \mu_{1}=i(2 \pi)^{6}\left(\bar{c}_{0} a_{1}-c_{1} \bar{a}_{0}-d_{1} \bar{b}_{0}\right), \\
\mu_{2}=(2 \pi)^{5}\left|d_{1}\right|^{2}, & \mu_{3}=i(2 \pi)^{6}\left(\bar{c}_{1} a_{1}-\bar{a}_{1} c_{1}+\bar{d}_{1} b_{1}-\bar{b}_{1} d_{1}\right) .
\end{array}
$$


One finds the following expression for the Kähler metric

$$
g_{x \bar{x}}=-\frac{\mu_{2}}{\mu_{0}} \ln |x|^{2}+\left(\frac{\left|\mu_{1}\right|^{2}}{\mu_{0}^{2}}-\frac{2 \mu_{2}+\mu_{3}}{\mu_{0}}\right)+O(|x| \ln |x|) .
$$

Then the curvature form is

$$
R_{x \bar{x}}=\frac{1}{4|x|^{2}} \frac{1}{(\ln |x|+C)^{2}},
$$

where the constant $C$ is determined to be

$$
C=1-\frac{\left|\mu_{1}\right|^{2}}{2 \mu_{0} \mu_{2}}+\frac{\mu_{3}}{2 \mu_{2}} \approx-0.738 .
$$

In computing Kähler covariantized derivatives with respect to $\psi$, it is also useful to note that

$$
\partial_{x} K_{\psi}=-\frac{\mu_{1}}{\mu_{0}}-\frac{\mu_{2}}{\mu_{0}} \bar{x} \ln |x|^{2}+O(x) .
$$

\subsection{Distribution of flux vacua}

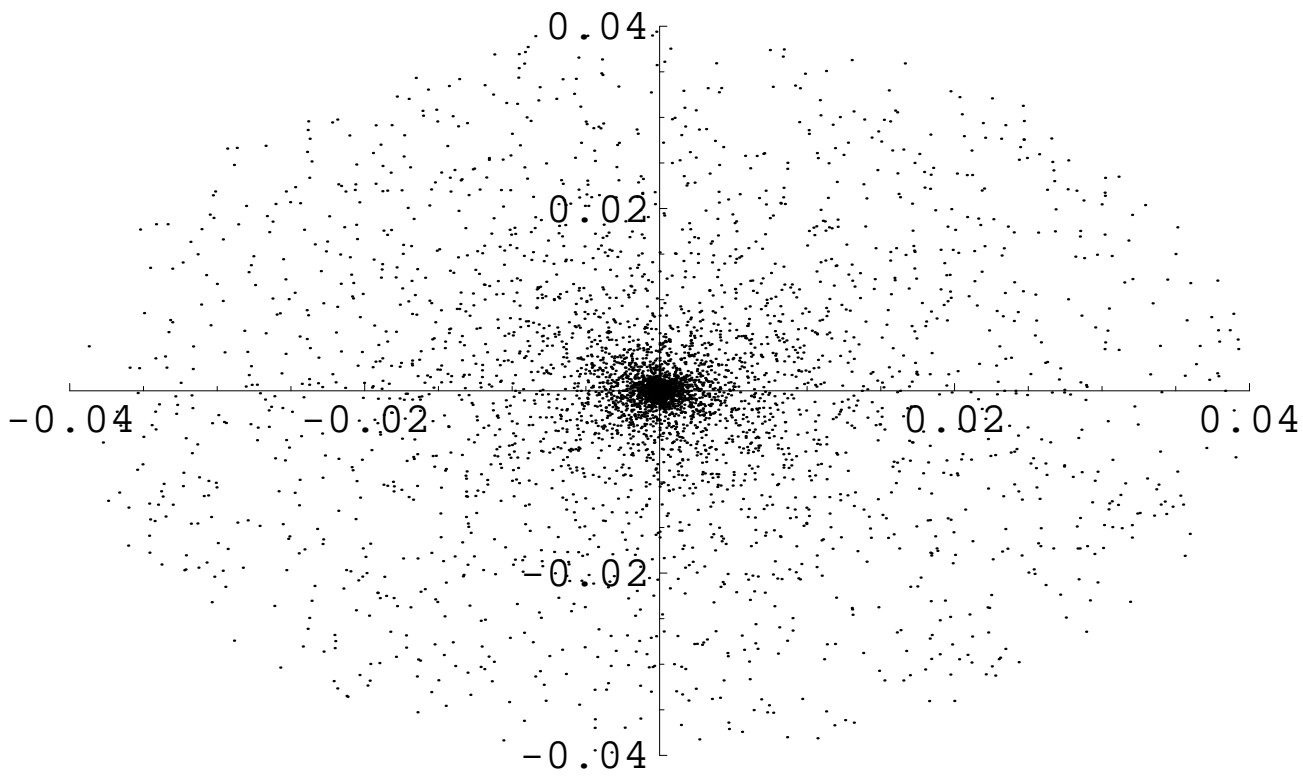

Fig. 3: Each point is a vacuum on the $x=1-\psi$ complex plane. The monte carlo simulation data is: number of random fluxes $N=5 \times 10^{7}$; random flux interval $f, h \in(-100,100)$; complex structure $\psi$ space region $|x|<0.04$. There are 11249 vacua, but 6306 of them arise at $|x|<.00001$ and have been removed from the plot (they would all cluster at the origin). 


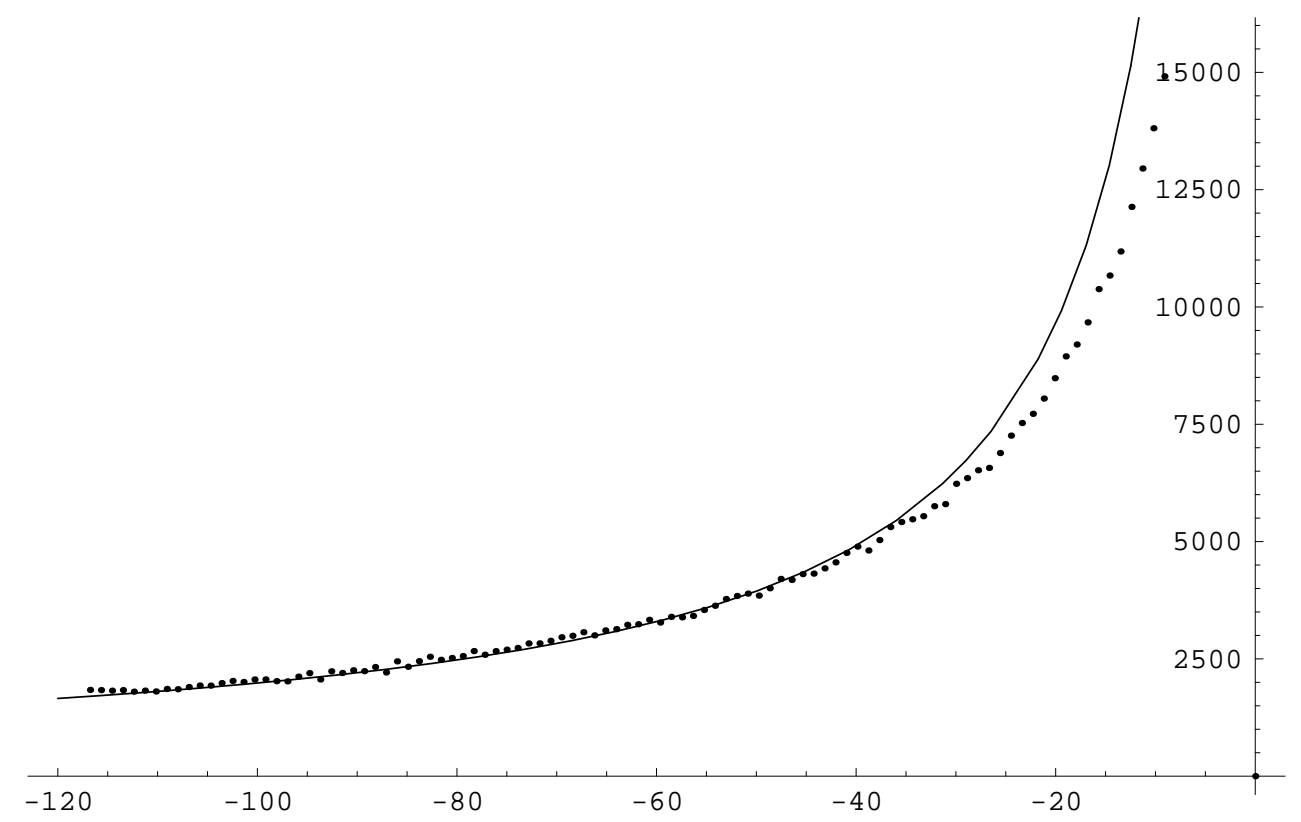

Fig. 4: The plot of a numerical evaluation of $\int_{\mathcal{M}} \operatorname{det}(-R-\omega)$. The monte carlo simulation data for each point is: number of random fluxes $N=10^{7}$; random flux interval $f, h \in(-60,60)$. The data is fit by the curve $-\frac{200000}{\rho+C}$, where $\rho=\ln r$. The conifold point $r=0$ is at $\rho=-\infty$ for this coordinate.

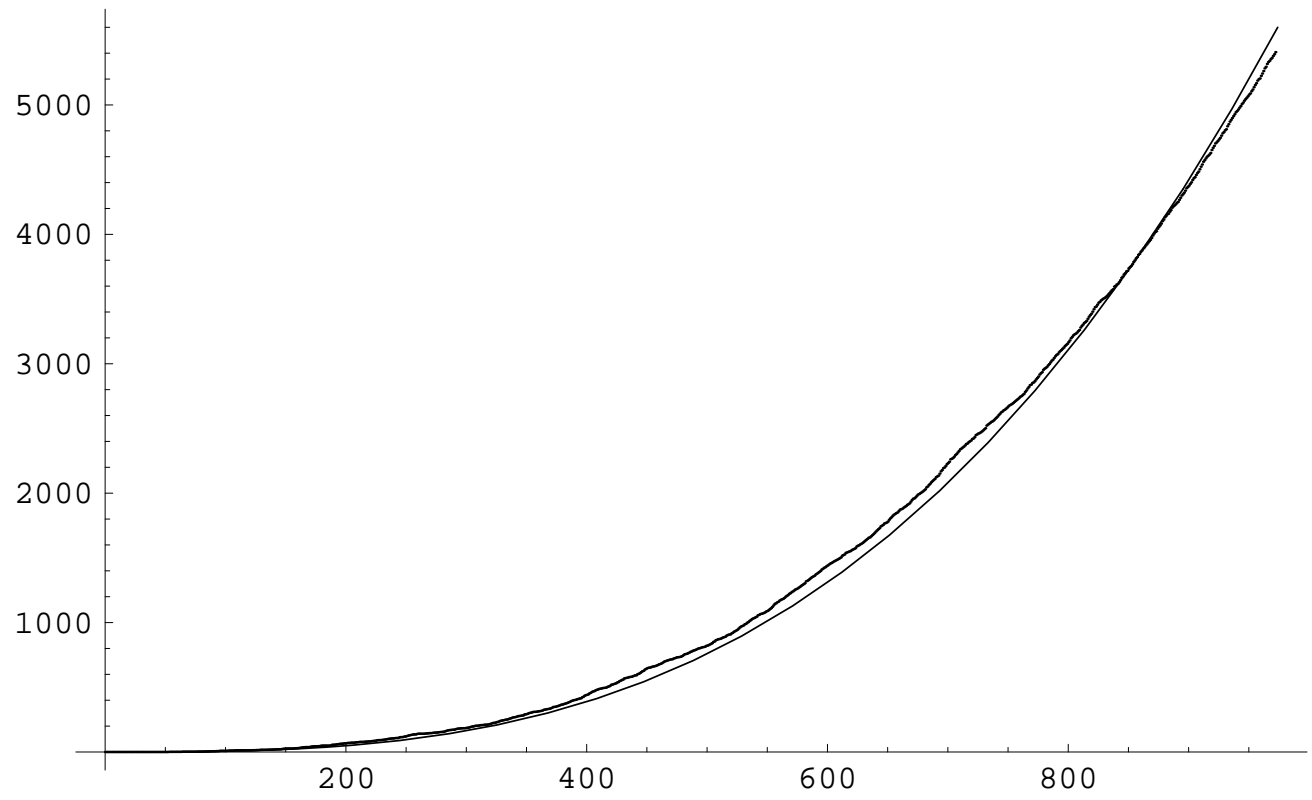

Fig. 5: The numerical results for the number of vacua with $N_{\text {flux }}<L$ for $L \in$ $(1,972)$. The monte carlo simulation data is: number of random fluxes $N=10^{10}$; random flux interval $f, h \in(-200,200)$; complex structure $\psi$ space region $|x|<$ 0.001 . The data is fit by the curve $\frac{L^{3}}{165000}$. 
We numerically solved for vacua around $\psi=1$. To compare with the prediction from $\S 2.1$, we need to determine the curvature and Kähler form of the moduli space. Using the results of $\S 5.1$, we find (including only the leading contribution coming from the curvature form)

$$
\int_{\mathcal{M}} \operatorname{det}(-R-\omega)=\text { const } \int_{0}^{r} \frac{1}{|x|^{2}} \frac{1}{(\ln |x|+C)^{2}}|x| d|x|=-\frac{\text { const }}{\ln r+C}
$$

for the portion of the fundamental region of complex structure moduli space $\mathcal{M}:|x|<r$.

The equations for flux vacua take the form

$$
\begin{gathered}
D_{\tau} W=0 \Rightarrow \tau=\frac{f \cdot \Pi^{\dagger}}{h \cdot \Pi^{\dagger}}=\frac{f_{1} \bar{a}_{0}+f_{2} \bar{b}_{0}+f_{3} \bar{c}_{0}}{h_{1} \bar{a}_{0}+h_{2} \bar{b}_{0}+h_{3} \bar{c}_{0}}+O(|x| \ln |x|) ; \\
D_{\psi} W=0 \Rightarrow \ln (x)=-\frac{2 \pi i}{d_{1}}\left[\frac{\left(f_{1}-\tau h_{1}\right)\left(a_{1}-\frac{\mu_{1}}{\mu_{0}} a_{0}\right)+\left(f_{2}-\tau h_{2}\right)\left(b_{1}-\frac{\mu_{1}}{\mu_{0}} b_{0}\right)}{f_{2}-\tau h_{2}}+\right. \\
\left.+\frac{\left(f_{3}-\tau h_{3}\right)\left(c_{1}-\frac{\mu_{1}}{\mu_{0}} c_{0}\right)+\left(f_{4}-\tau h_{4}\right) d_{1}}{f_{2}-\tau h_{2}}\right]-1 .
\end{gathered}
$$

We performed a monte carlo simulation, solving the equations of motion for randomly selected fluxes. In Figure 3, we plot the distribution of vacua near $\psi=1$ on the $x=1-\psi$ plane. The most striking thing about Figure 3 is the clustering of vacua around the conifold point [19,33]. This would be expected from the curvature dependence of the result for the index (2.1) (which, as shown in Figure 4, is in good agreement with our results). This is interesting both because it shows that the conjecture (2.1) contains much more information than a plausible boring conjecture like "vacua are uniformly distributed on the moduli space," and that furthermore the detailed distribution we find experimentally is in fact captured by the simple formula (2.1)!

As described in [4, 13], the conifold point is particularly interesting because it provides a natural mechanism for flux-generated hierarchies (as in the noncompact KlebanovStrassler solution [34]), and can provide small numbers which help with moduli stabilization or attaining small vacuum energy. It could therefore also be physically important that it dominates the distribution in Figure 3. Flux vacua near the conifold [4] (see also [35]) can explain light scalars by warping (instead of protecting their masses by supersymmetry). Therefore, if this feature persisted in the full problem of characterizing the string landscape, it could mean that the correct stringy notion of naturalness allows warping as a natural explanation for light scalars (as in Randall-Sundrum scenarios [36]), in addition to 
low-energy supersymmetry. Of course this is mere speculation at this point, but indicates the kinds of lessons one could hope to learn.

As shown in Fig. 5, for the range of $L$ most relevant to Model A of [7], we again seem to obtain close to $L^{3}$ scaling for the number of vacua in this region of moduli space. It is quite possible that at much larger $L$, this behavior would be modified to $L^{4}$ in accord with (2.6), and that a more detailed theory could explain the $L^{3}$ scaling visible here.

\section{Conclusions}

In this paper, we have seen that very simple conjectures can serve to capture the detailed structure of the numbers and distribution of flux vacua on Calabi-Yau moduli spaces. While the flux potentials are surely not the full story in string theory, they already encapsulate very rich behavior (including moduli stabilization, warping, supersymmetry breaking, etc.), and may exhibit much of the complexity of the full story. It is therefore heartening that simple conjectures like those of $\S 2$ can essentially classify such large ensembles of vacua in simple terms. This increases our confidence that somewhere down the line, similar simple conjectures which capture basic aspects of the structure of the full space of string vacua may become available.

The most interesting physical feature to emerge is the existence of special loci in the moduli space which serve as attractors, in the sense that a large fraction of the flux vacua cluster around them. In the one-dimensional moduli space we examined here, the conifold plays this role, in a way that is immediately apparent from Figure 3. The existence of such attractors in a more general setting could be important in helping to define a stringy notion of naturalness.

A useful generalization of this work would involve the study of models with many complex structure moduli. Finding concrete models which meet the requirements of [13] or its generalizations should also become possible, as we gain the ability to scan over large sets at once. 3 The ensemble where one focuses on IIB flux superpotentials could also be naturally enlarged to include generic Non-Kähler type II models [38], or classes of vacua where one includes an analysis of nontrivial D-brane worldvolume superpotentials (see 39] for a recent review). This could be important given that D-brane theories in flux

3 After this paper was completed, the interesting work [37] appeared where explicit examples along the lines of [13] are presented, including Kähler modulus stabilization. 
backgrounds can give rise to very large numbers of vacua (see e.g. [40,14]). And finally, it would be valuable to study other well-motivated toy ensembles which characterize the sets of supergravities emerging from others limits of M-theory, like the heterotic theory.

\section{Acknowledgements}

We are very grateful to S. Trivedi for early collaboration and for sharing his insights, and to M. Douglas, S. Shenker, E. Silverstein and L. Susskind for helpful discussions about the statistics of flux vacua. S.K. would like to thank G. Dvali and especially S. Dimopoulos for many interesting conversations about the "attractive" nature of the conifold point and possible physical implications. P.K.T. is very much thankful to the HETG group at Harvard University, especially to S. Minwalla, for kind hospitality at Harvard and for providing a lot of encouragement. He also thanks all the people of India for enthusiastically supporting research in String Theory. The work of A.G. was partially supported by РФФИ grant 02-01-00695 and INTAS 03-51-6346. The work of S.K. was supported in part by a David and Lucile Packard Foundation Fellowship for Science and Engineering, NSF grant PHY-0097915, and the DOE under contract DE-AC03-76SF00515. The work of P.K.T. was partially supported by Harvard University and TIFR. 


\section{References}

[1] P. Candelas, G. Horowitz, A. Strominger and E. Witten, "Vacuum Configurations for Superstrings," Nucl. Phys. B258 (1985) 46.

[2] A. Strominger, "Superstrings with Torsion," Nucl. Phys. B274 (1986) 253;

J. Polchinski and A. Strominger, "New vacua for type II string theory," Phys. Lett. B388 (1996) 736, hep-th/9510227;

K. Becker and M. Becker, "M-theory on eight manifolds," Nucl. Phys. B477 (1996) 155, hep-th/9605053;

J.Michelson, "Compactifications of type IIB strings to four-dimensions with non-trivial classical potential," Nucl. Phys. B495 (1997) 127, hep-th/9610151;

K. Dasgupta, G. Rajesh and S. Sethi, "M-theory, orientifolds and G-flux," JHEP 9908 (1999) 023, hep-th/9908088;

B. Greene, K. Schalm and G. Shiu, "Warped compactifications in M and F theory," Nucl. Phys. B584 (2000) 480, hep-th/0004103;

G. Curio, A. Klemm, D. Lüst and S. Theisen, "On the Vacuum Structure of Type II String Compactifications on Calabi-Yau Spaces with H-Fluxes," Nucl. Phys. B609 (2001) 3, hep-th/0012213;

K. Becker and M. Becker, "Supersymmetry Breaking, M Theory and Fluxes," JHEP 010 (2001) 038, hep-th/0107044;

M. Haack and J. Louis, "M theory compactified on Calabi-Yau fourfolds with background flux," Phys. Lett. B507 (2001) 296, hep-th/0103068;

J. Louis and A. Micu, "Type II theories compactified on Calabi-Yau threefolds in the presence of background fluxes," Nucl.Phys. B635 (2002) 395, hep-th/0202168.

[3] S. Gukov, C. Vafa and E. Witten, "CFTs from Calabi-Yau Fourfolds," Nucl. Phys. B584 (2000) 69, hep-th/9906070;

T.R. Taylor and C. Vafa, "RR flux on Calabi-Yau and partial supersymmetry breaking," Phys. Lett. B474 (2000) 130, hep-th/9912152;

P. Mayr, "On Supersymmetry Breaking in String Theory and its Realization in Brane Worlds," Nucl. Phys. B593 (2001) 99, hep-th/0003198.

[4] S. Giddings, S. Kachru, and J. Polchinski, "Hierarchies from Fluxes in String Compactifications," Phys. Rev. D66 (2002) 106006, hep-th/0105097.

[5] S. Kachru, M. Schulz and S. P. Trivedi, "Moduli Stabilization from Fluxes in a Simple IIB Orientifold," JHEP 0310 (2003) 007, hep-th/0201028;

A. Frey and J. Polchinski, "N=3 Warped Compactifications," Phys. Rev. D65 (2002) 126009, hep-th/0201029.

[6] P. Tripathy and S. P. Trivedi, "Compactification with Flux on K3 and Tori," JHEP 0303 (2003) 028, hep-th/0301139. 
[7] A. Giryavets, S. Kachru, P. Tripathy and S. Trivedi, "Flux Compactifications on Calabi-Yau Threefolds," JHEP 0404 (2004) 003, hep-th/0312104.

[8] O. DeWolfe and S. Giddings, "Scales and hierarchies in warped compactifications and brane worlds," Phys. Rev. D67 (2003) 066008, hep-th/0208123.

[9] J. Cascales, M. Garcia de Moral, F. Quevedo and A. Uranga, "Realistic D-brane Models on Warped Throats: Fluxes, Hierarchies and Moduli Stabilization," hepth/0312051;

J. Cascales and A. Uranga, "Chiral 4d String Vacua with D-branes and Moduli Stabilization," hep-th/0311250;

J. Cascales and A. Uranga, "Chiral $4 \mathrm{~d} \mathcal{N}=1$ String Vacua with D-Branes and NSNS and RR Fluxes," JHEP 0305 (2003) 011, hep-th/0303024;

R. Blumenhagen, D. Lüst and T. Taylor, "Moduli Stabilization in Chiral Type IIB Orientifold Models with Fluxes," Nucl. Phys. B663 (2003) 219, hep-th/0303016.

[10] R. D'Auria, S. Ferrara and S. Vaula, " $\mathcal{N}=4$ gauged supergravity and a IIB orientifold with fluxes," New J. Phys. 4 (2002) 71, hep-th/0206241;

S. Ferrara and M. Porrati, "N $\mathcal{N}=1$ no-scale supergravity from IIB orientifolds," Phys. Lett. B545 (2002) 411, hep-th/0207135;

R. D'Auria, S. Ferrara, M. Lledo and S. Vaula, "No-scale $\mathcal{N}=4$ supergravity coupled to Yang-Mills: the scalar potential and super-Higgs effect," Phys. Lett. B557 (2003) 278, hep-th/0211027;

R. D'Auria, S. Ferrara, F. Gargiulo, M. Trigiante and S. Vaula, " $\mathcal{N}=4$ supergravity Lagrangian for type IIB on $T^{6} / Z_{2}$ orientifold in presence of fluxes and D3 branes," JHEP 0306 (2003) 045, hep-th/0303049;

L. Andrianopoli, S. Ferrara and M. Trigiante, "Fluxes, supersymmetry breaking and gauged supergravity," hep-th/0307139;

B. de Wit, Henning Samtleben and M. Trigiante, "Maximal Supergravity from IIB Flux Compactifications," hep-th/0311224.

[11] P.G. Camara, L.E. Ibanez and A.M Uranga, "Flux Induced SUSY Breaking Soft Terms, hep-th/0311241;

M. Grana, T. Grimm, H. Jockers and J. Louis, "Soft Supersymmetry Breaking in Calabi-Yau Orientifolds with D-branes and Fluxes," hep-th/0312232;

A. Lawrence and J. McGreevy, "Local String Models of Soft Supersymmetry Breaking," hep-th/0401034.

[12] P. Candelas, X. de la Ossa, P. Green and L. Parkes, "A Pair of Calabi-Yau Manifolds as an Exactly Soluble Superconformal Theory," Nucl. Phys. B359 (1991) 21.

[13] S. Kachru, R. Kallosh, A. Linde and S.P. Trivedi, "de Sitter Vacua in String Theory," Phys. Rev. D68 (2003) 046005, hep-th/0301240.

[14] M. Douglas, "The Statistics of String/M-theory Vacua," JHEP 0305 (2003) 046, hepth/0303194. 
[15] R. Bousso and J. Polchinski, "Quantization of Four-Form Fluxes and Dynamical Neutralization of the Cosmological Constant," JHEP 0006 (2000) 006, hep-th/0004134.

[16] J. Feng, J. March-Russell, S. Sethi and F. Wilczek, "Saltatory Relaxation of the Cosmological Constant," Nucl. Phys. B602 (2001) 307, hep-th/0005276.

[17] S. Ashok and M. Douglas, "Counting Flux Vacua," JHEP 0401 (2004) 060, hepth/0307049.

[18] M. Douglas, B. Shiffman and S. Zelditch, "Critical Points and Supersymmetric Vacua," arXiv:math.CV/0402326.

[19] F. Denef and M. Douglas, "Distributions of Flux Vacua," hep-th/0404116.

[20] L. Susskind, "The Anthropic Landscape of String Theory," hep-th/0302219.

[21] T. Banks, M. Dine and E. Gorbatov, "Is there a string theory landscape?," hepth/0309170.

[22] A. Saltman and E. Silverstein, "The Scaling of the No-Scale Potential and de Sitter Model Building," hep-th/0402135;

C.P. Burgess, R. Kallosh and F. Quevedo, "De Sitter Vacua from Supersymmetric D-terms," JHEP 0310 (2003) 056, hep-th/0309187;

C. Escoda, M. Gomez-Reino and F. Quevedo, "Saltatory de Sitter String Vacua," JHEP 0311 (2003) 065, hep-th/0307160;

A. Frey, M. Lippert and B. Williams, "The Fall of Stringy de Sitter," Phys. Rev. D68 (2003) 046008, hep-th/0305018.

[23] M. Becker, G. Curio and A. Krause, "De Sitter Vacua from Heterotic M Theory," hep-th/0403027;

R. Brustein and S.P. de Alwis, "Moduli Potentials in String Compactifications with Fluxes: Mapping the Discretuum," hep-th/0402088;

S. Gukov, S. Kachru, X. Liu and L. McAllister, "Heterotic Moduli Stabilization with Fractional Chern-Simons Invariants," hep-th/0310159;

E. Buchbinder and B. Ovrut, "Vacuum Stability in Heterotic M-theory," hepth/0310112.

[24] A. Maloney, E. Silverstein and A. Strominger, "De Sitter Space in Noncritical String Theory," hep-th/0205316.

[25] For interesting work in this direction with further references, see: G.L. Cardoso, G. Curio, G. Dall'Agata, D. Lüst, P. Manousselis and G. Zoupanos, "Nonkahler String Backgrounds and their Five Torsion Classes," Nucl. Phys. B652 (2003) 5, hep-th/0211118; K. Becker, M. Becker, K. Dasgupta and P. Green, "Compactifications of Heterotic Theory on NonKähler Complex Manifolds I," JHEP 0304 (2003) 007, hep-th/0301161;

K. Becker, M. Becker, K. Dasgupta, P. Green, E. Sharpe, "Compactifications of Heterotic Strings on NonKähler Complex Manifolds II," Nucl. Phys. B678 (2004) 19, hep-th/0310058; 
J. Gauntlett, D. Martelli and D. Waldram, "Superstrings with Intrinsic Torsion," Phys. Rev. D69 (2004) 086002, hep-th/0302158.

[26] G. Moore, "Les Houches Lectures on Strings and Arithmetic," hep-th/0401049.

[27] S. Kachru, J. Pearson and H. Verlinde, "Brane/Flux Annihilation and the String Dual of a Non-supersymmetric Field Theory," JHEP 0206 (2002) 021, hep-th/0112197.

[28] S. Kachru, unpublished; E. Silverstein, unpublished; L. Susskind, unpublished.

[29] A. Sen, "Orientifold limit of F-theory vacua," Phys. Rev. D55 (1997) 7345, hepth/9702165.

[30] S. Sethi, C. Vafa and E. Witten, "Constraints on Low-Dimensional String Compactifications," Nucl. Phys. B480 (1996) 213, hep-th/9606122.

[31] B. Greene and R. Plesser, "Duality in Calabi-Yau Moduli Space," Nucl. Phys. B338 (1990) 15.

[32] A. Klemm and S. Theisen, "Considerations of One-Modulus Calabi-Yau Compactifications: Picard-Fuchs Equations, Kähler Potentials and Mirror Maps," Nucl.Phys. B389 (1993) 153, hep-th/9205041.

[33] M. Douglas, various talks, Fall 2003.

[34] I. Klebanov and M. Strassler, "Supergravity and a Confining Gauge Theory: Duality Cascades and $\chi$ SB Resolution of Naked Singularities," JHEP 008 (2000) 052, hepth/0007191.

[35] H. Verlinde, "Holography and Compactification," Nucl. Phys. B580 (2000) 264, hepth/9906182;

C. Chan, P. Paul and H. Verlinde, "A Note on Warped String Compactification," Nucl. Phys. B581 (2000) 156, hep-th/0003236.

[36] L. Randall and R. Sundrum, " A Large Mass Hierarchy from a Small Extra Dimension," Phys. Rev. Lett. 83 (1999) 3370, hep-th/9905221.

[37] F. Denef, M. Douglas and B. Florea, "Building a better racetrack," hep-th/0404257.

[38] S. Gurrieri, J. Louis, A. Micu and D. Waldram, "Mirror Symmetry in Generalized Calabi-Yau Compactifications," Nucl. Phys. B654 (2003) 61, hep-th/0211102;

S. Kachru, M. Schulz, P. Tripathy and S. Trivedi, "New Supersymmetric String Compactifications," JHEP 0303 (2003) 061, hep-th/0211182.

[39] W. Lerche, "Special Geometry and Mirror Symmetry for Open String Backgrounds with $\mathcal{N}=1$ Supersymmetry," hep-th/0312326.

[40] J. Polchinski and M. Strassler, "The String Dual of a Confining Four-Dimensional Gauge Theory," hep-th/0003136. 